\title{
Nutrition Knowledge and Attitudes of Pre-registered and Registered Nurses in relation to Cancer Survivorship
}

\author{
S. R. Rodman and J. L. Murphy \\ School of Health and Social Care, Bournemouth University, Bournemouth, BHI 3LT, UK
}

A greater focus on recovery, health and well-being after cancer treatment including advice on diet and lifestyle changes as part of personalised care planning is a key aspect of the National Cancer Survivorship Initiative ${ }^{(1)}$. Front-line staff are in a prime position, so it is vital that they can confidently provide accurate and consistent information on promoting healthy lifestyles. Previous studies have shown the nutrition knowledge of both pre-registered and registered nurses is poor, notably in relation to obesity and health ${ }^{(2,3)}$, but little is known of their perceived knowledge and understanding of diet and lifestyle change in relation to cancer survivorship.

The current study aimed to assess the nutrition knowledge and attitudes of nurses with respect to their level of education and the provision of advice and support for cancer survivors. A modification of a validated questionnaire ${ }^{(4)}$ that included questions on the World Cancer Research Fund recommendations ${ }^{(5)}$ was emailed to both pre-registered and registered nurses at Bournemouth University. Results from 102 respondents are shown in the table.

\begin{tabular}{lcc}
\hline & $\begin{array}{c}\text { Mean knowledge score } \\
\text { Maximum achievable score }=145 \\
\text { (Min-Max) }\end{array}$ & $\begin{array}{c}\text { Mean }(\% \text { of maximum) } \\
\text { (Min-Max) }\end{array}$ \\
\hline Pre-registered Nurses $(n$ 84) & $81(35-115)$ & $56(24-79)$ \\
Registered Nurses $(n$ 18$)$ & $98^{*}(84-117)$ & $68(58-81)$ \\
\hline
\end{tabular}

*Mean value was significantly different from Pre-registered nurses $P<0.01$.

The overall knowledge score was significantly greater for the registered than pre-registered nurses $(P<0.01)$ although there was a wide difference in the scores observed between the pre-registered nurses. There was a positive association between age and total knowledge score $(r 0.57 P<0.01)$. No difference was observed between level of education and total knowledge score. The link between diet and cancer was cited by $28 \%$ of respondents and only $34 \%$ chose the correct dietary recommendations for cancer survivors. Attitudinal questions found nurses to be either occasionally confident when giving nutrition advice (55\%) or confident most of the time (40\%). The source of their nutritional information was cited as early-life influences $(71 \%)$ and the media (66\%). The majority (70\%) of all respondents would like further training and of these, more than half felt that using a combination of face-to-face interaction and e-learning was the best approach to provide training.

Overall these findings suggest that the general awareness of the importance of diet and lifestyle issues in relation to cancer survivorship is limited. Further attention needs to be directed towards providing nutrition education and training for nurses from the evidence base. Further research is needed to understand the best approach to deliver this training at pre-registered level and as part of continued professional development.

1. Department of Health (2010) The National Cancer Survivorship Initiative Vision. London: Department of Health.

2. Hankey CR, Eley S, Leslie WS et al. (2004) Public Health Nutr 7, 337-343.

3. Swift JA, Sheard C \& Rutherford M (2007) J Hum Nutr Diet 20, 599-604.

4. Parmenter K \& Wardle J (1999) Eur J Clin Nutr 53, 298-308.

5. World Cancer Research Fund (2007) Food, Nutrition, Physical Activity, and the Prevention of Cancer: a Global Perspective. Washington DC: World Cancer Research Fund and American Institute for Cancer Research. 